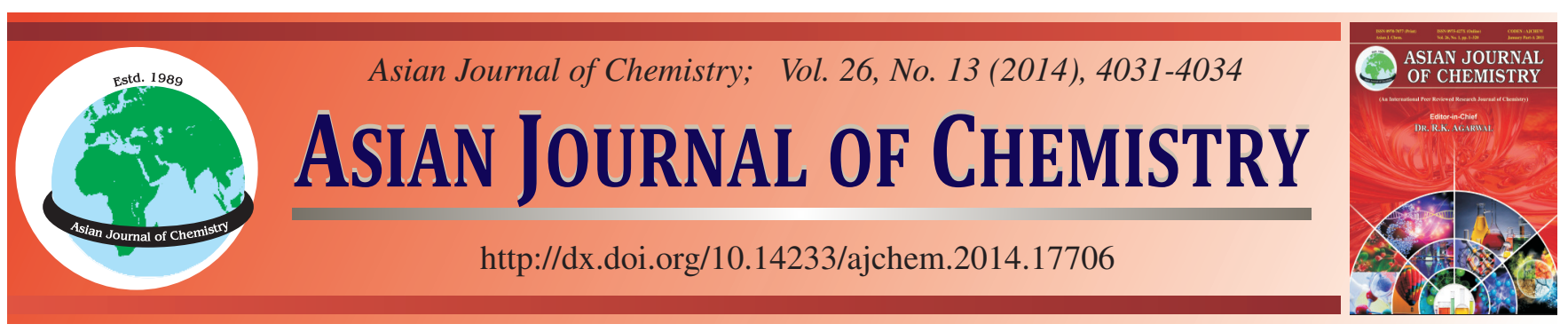

\title{
Hyperbranched Polyglycerol-Coated Magnetic Nanoparticles as Draw Solute in Forward Osmosis $\dagger$
}

Hee-Man Yang*, Bum-Kyoung Seo, Kune-Woo Lee and Jei-Kwon Moon

Decontamination \& Decommissioning Research Division, Korea Atomic Energy Research Institute, Yuseong-gu, Daejeon 305-353, Republic of Korea

*Corresponding author: Fax: +82 42 8688667; E-mail: hmyang@ kaeri.re.kr

Published online: 23 June 2014;

AJC-15401

\begin{abstract}
Water soluble hyperbranched polyglycerol-coated magnetic nanoparticles (HPG-MNPs) were introduced as a new draw solute in forward osmosis (FO) to prevent their aggregate for better forward osmosis performance. The HPG-MNPs were successfully synthesized by grafting polyglycerol onto the surfaces of magnetite through a ring-opening polymerization of glycidol. The HPG-MNPs exhibit excellent dispersibility in aqueous solution and the highest water flux achieved in a forward osmosis process was $6.7 \mathrm{~L} / \mathrm{m}^{2} \mathrm{~h}$ for HPG-MNPs when deionized water is used as the feed solution.
\end{abstract}

Keywords: Polyglycerol, Magnetite nanoparticles, Draw solute, Forward osmosis.

\section{INTRODUCTION}

Forward osmosis (FO), a low energy technique based on membrane technologies, has recently garnered attention for its utility in wastewater treatment and desalination applications. In the forward osmosis process, water flows across a semipermeable membrane from a solution with a low osmotic pressure (the feed solution) to a solution with a high osmotic pressure (the draw solution) $)^{1}$. The driving force in forward osmosis processes is provided by the osmotic gradient between the two solutions. Low energy costs and low degrees of membrane fouling are two of the advantages conveyed by forward osmosis processes over other processes, such as reverse osmosis processes that rely on a hydraulic pressure driving force $^{1,2}$.

Many efforts have been made to develop a feasible draw solute having high osmotic pressure in solution for high water flux to improve the performances of forward osmosis processes. Among the variety of draw solutions such as inorganic salt, fertilizer, saccharide and polyelectrolyte, inorganic salt has the majority of forward osmosis studies and they are still extensively utilized nowadays. However, most draw solutes are difficult to separate from the draw solution for reuse and also have loss problem of draw solutes caused by reverse diffusion ${ }^{3}$. Chung et al. recently reported the use of hydrophilic linear polymer coated magnetic nanoparticles (MNPs) as a draw solute and showed the advantages of low reverse flux compared to traditional draw solute ${ }^{4,5}$. Moreover, these MNPs can be recovered by applying a magnetic field. However, they are found to aggregate as larger sizes under a high strength magnetic field, causing a significant decrease in forward osmosis performance.

In this study, we introduced highly water-soluble hyperbranched polyglycerol-coated magnetic nanoparticles (HPGMNPs) as draw solutes to prevent the MNPs aggregate by high strength magnetic field. It is known that the highly branched, globular architecture of HPG significantly increase solubility compared to linear polymer and they are eco-friendly ${ }^{6}$. After synthesis of HPG-MNPs, the physical properties of resulting HPG-MNPs and their performance as a draw solute in forward osmosis processes were characterized.

\section{EXPERIMENTAL}

Synthesis of magnetic nanoparticles: Iron acetylacetonate ( $3 \mathrm{mmol}$ ) and triethylene glycol (TREG, $60 \mathrm{~mL}$ ) were mixed and heated to reflux for $3 \mathrm{~h}$ under $\mathrm{N}_{2}$ atmosphere. After cooling to room temperature, ethyl acetate was added to the reaction solution to yield a black precipitate comprising MNPs. The nanoparticles were collected from the solution upon application of an external magnetic field. After washing with ethyl acetate three times, the purified MNPs were obtained by drying the resulting precipitate under vacuum. 
Synthesis of hyperbranched polyglycerol coatedmagnetic nanoparticles: $300 \mathrm{mg}$ of the as-synthesized MNPs was mixed with $60 \mathrm{~mL}$ of glycidol under sonication for $1 \mathrm{~h}$. The mixture was then magnetically stirred at $140{ }^{\circ} \mathrm{C}$ under $\mathrm{N}_{2}$ atmosphere for $20 \mathrm{~h}$. The resulting gel was cooled to room temperature and diluted with distilled water and recovered by ultracentrifugation $(50,000 \mathrm{rpm}$ for $0.5 \mathrm{~h})$. The washing process was repeated at least three times to remove any by-products. The washed sample was subsequently lyophilized.

Forward osmosis (FO) process: Deionized water was prepared for use in the feed solutions. The draw solutions were prepared using various concentrations of the HPG-MNPs. The commercial forward osmosis membrane (HTI OsMem ${ }^{\mathrm{TM}}$ ) was prepared by coating cellulose triacetate (CTA) onto a polyester screen support. A forward osmosis membrane sheet with an effective membrane area of $5 \mathrm{~cm}(\mathrm{~W}) \times 10 \mathrm{~cm}(\mathrm{~L})$ was housed in a cross flow membrane cell. Rectangular-patterned stainless steel spacers were placed on either side of the membrane in such a way as to permit the feed and draw solutions to flow across the membrane. The initial volumes of the feed and draw solution tanks were $200 \mathrm{~mL}$. The cross flow velocities of the feed and draw solutions were fixed at 25 and $17 \mathrm{~cm} / \mathrm{s}$, with pressure of 8.5 and $5.5 \mathrm{psi}$, respectively. The cross flow velocity was calculated based on the ratio of the cross flow rate to the cross-section area of the flow channel, which was $5 \mathrm{~cm}(\mathrm{~W}) \times$ $0.2 \mathrm{~cm}$ (D). The feed and draw solution temperatures were maintained at $20^{\circ} \mathrm{C}$.

\section{RESULTS AND DISCUSSION}

The as-synthesized MNPs were prepared using the thermal decomposition method in a polyol medium comprising TREG without any surfactants, as reported previously ${ }^{7}$. X-Ray diffraction (XRD) analysis confirmed the crystalline natures of all nanoparticles (Fig. 1). All peaks agreed well with the XRD patterns reported for bulk magnetite (JCPDS 07-322), indicating that the as-synthesized MNPs consisted of a $\mathrm{Fe}_{3} \mathrm{O}_{4}$ phase.

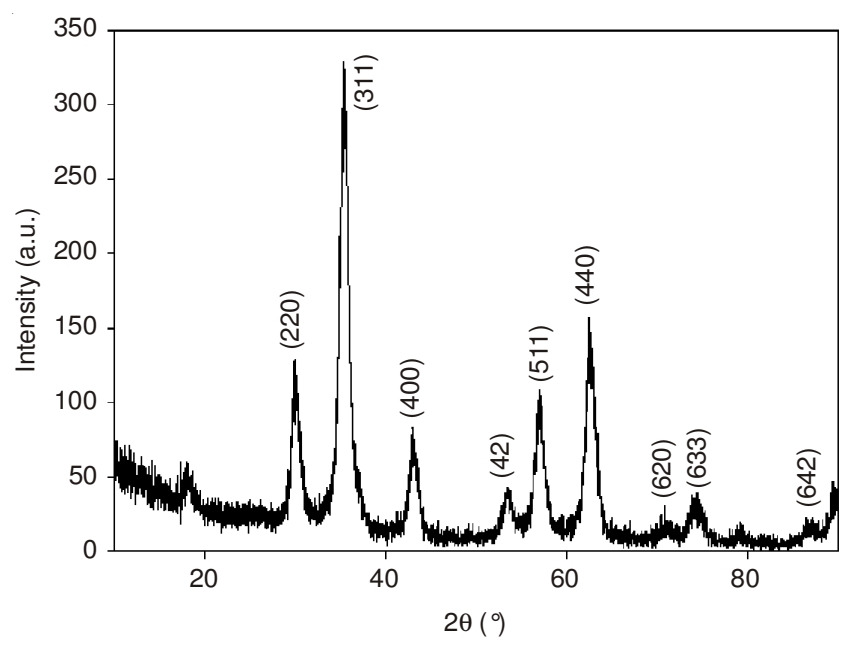

Fig. 1. X-Ray diffraction patterns of the as-synthesized $\mathrm{Fe}_{3} \mathrm{O}_{4} \mathrm{MNPs}$

The as-synthesized MNP morphology was confirmed by TEM imaging (Fig. 2(a)). The average diameters calculated from TEM image was $8.2 \pm 1.4 \mathrm{~nm}$. The average hydrodynamic diameter of the as-synthesized MNPs in water was measured

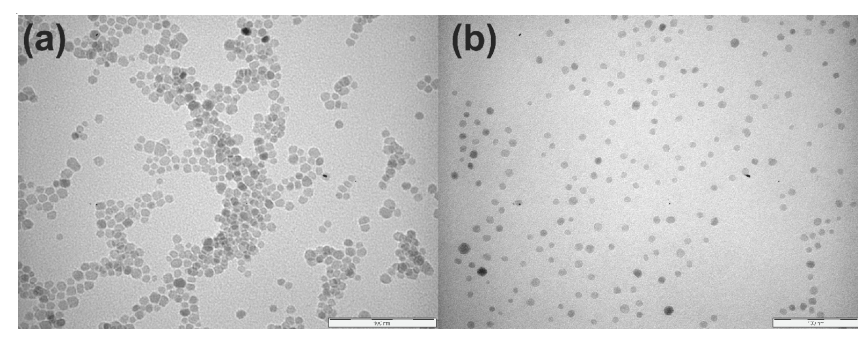

Fig. 2. TEM images of MNPs (a) the as-synthesized MNPs and (b) the HPG-MNPs. All scale bars indicate $100 \mathrm{~nm}$

by dynamic light scattering (DLS) to be $34.69 \pm 9.32 \mathrm{~nm}$. Although the as-synthesized MNPs were soluble in water and can be used as draw solute due to the presence of hydrophilic triethylene glycol, they are known to be aggregated when they are recovered by high strength magnetic field for reuse $e^{4}$.

To improve the water solubility of the MNPs and prevent the MNPs aggregation by high strength magnetic field, the as-synthesized MNPs were coated with HPG, which contains a large number of hydroxyl groups. The hydroxyl groups on the surfaces of the TREG-coated MNPs initiated HPG grafting through ring-opening polymerization of the glycidol groups on the MNP surfaces 8 .

Fig. 2(b) shows a TEM image of the HPG-MNPs. The nanocrystal cores of the HPG-MNPs were better dispersed than the as-synthesized MNPs (based on the TEM images, Fig. 2(a)), indicating that polymerization of the glycidol to form the HPG-MNPs enhanced the water solubility of the nanoparticles. The hydrodynamic diameter of the HPG-MNPs in water, measured by DLS, was $20.92 \pm 5.65 \mathrm{~nm}$, which was smaller than the value for the as-synthesized MNPs. The smaller value was attributed to the high water solubility of HPG-MNPs after grafting of HPG onto the single as-synthesized MNP. Furthermore, the HPG-MNPs displayed good stability in water, even after several lyophilization steps.

Fig. 3 showed the size of distribution (a) as synthesized MNPs and (b) the HPG-MNPs in water.

Fourier transform infrared (FTIR) analysis was used to explore the chemical structures of the as-synthesized MNPs (Fig. 4(a)) and HPG-MNPs (Fig. 4(b)). Fig. 4(a) shows the characteristic peaks of triethylene glycol at 1655, 1437 and $1069 \mathrm{~cm}^{-1}$ due to the $\mathrm{O}-\mathrm{H}$ stretching vibration, $\mathrm{C}-\mathrm{H}$ bending vibration and C-O bending vibration, respectively ${ }^{9}$. Fig. 4(b) shows that large increases in the absorption bands at 3400 , 2895 and $1090 \mathrm{~cm}^{-1}$ were observed in the HPG-MNPs. These bands were attributed to the large number of surface hydroxyl groups, $\mathrm{C}-\mathrm{H}$ stretching vibrations and the $\mathrm{C}-\mathrm{O}-\mathrm{C}$ ether stretches, respectively ${ }^{8}$. These results indicated that the HPG were successfully synthesized on the nanoparticles surfaces via ring-opening polymerization of glycidol.

The values of the wt $\% \mathrm{Fe}_{3} \mathrm{O}_{4}$ in the as-synthesized MNPs and HPG-MNPs, measured by thermal gravimetric analysis were 82.91 and $32.55 \%$, respectively (Fig. 5(a)). The TGA results of the as-synthesized MNPs were used to calculate the wt \% HPG in the HPG-MNPs: $60.74 \%$. The magnetic properties of the as-synthesized MNPs and HPG-MNPs were also examined using a vibrating sample magnetometer (VSM) heated to $300 \mathrm{~K}$ (Fig. 5(b)). Both of samples exhibited superparamagnetic behaviours. The saturated magnetization (Ms 

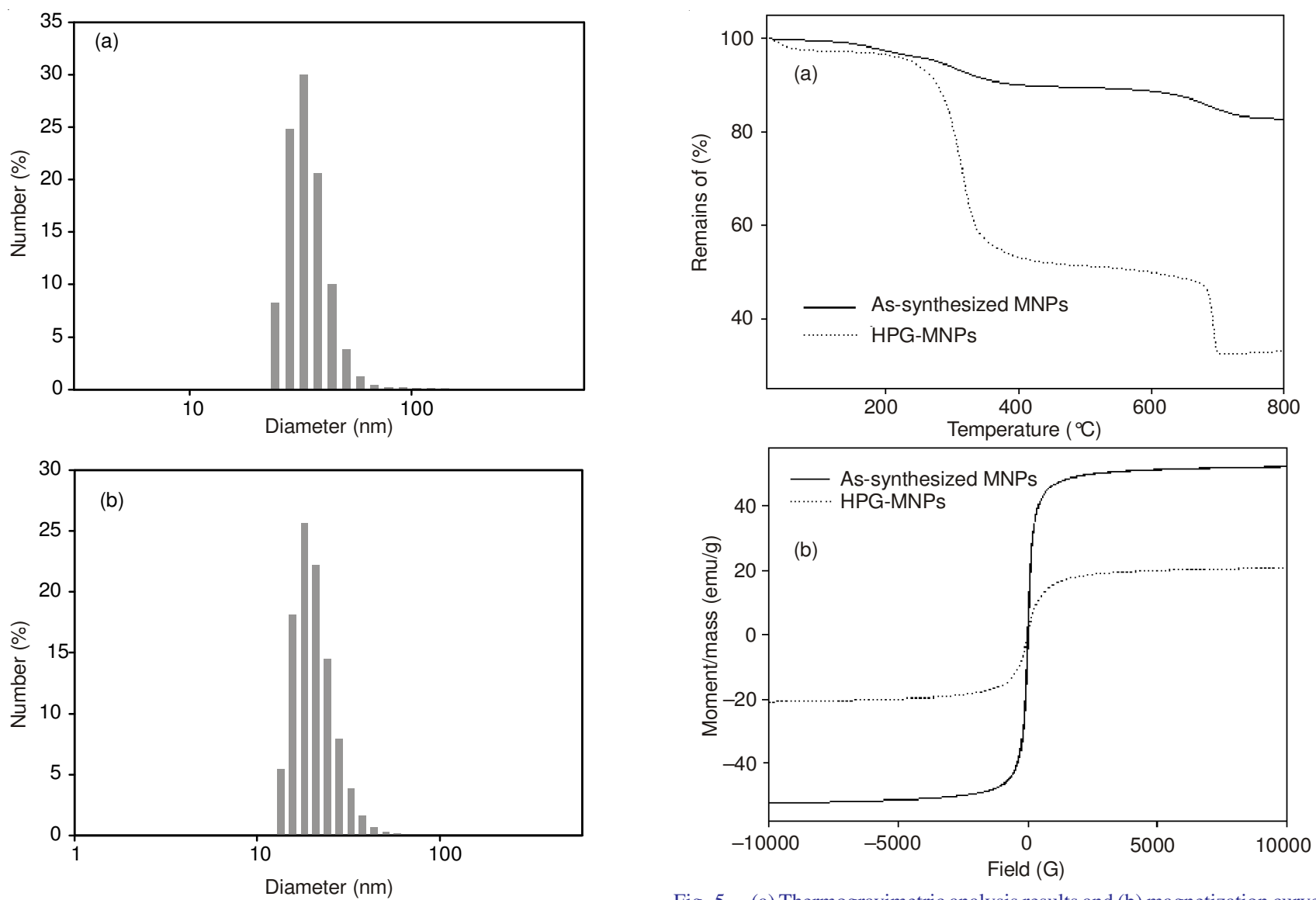

Fig. 3. Size distributions of (a) the as-synthesized MNPs and (b) the HPGMNPs, in water
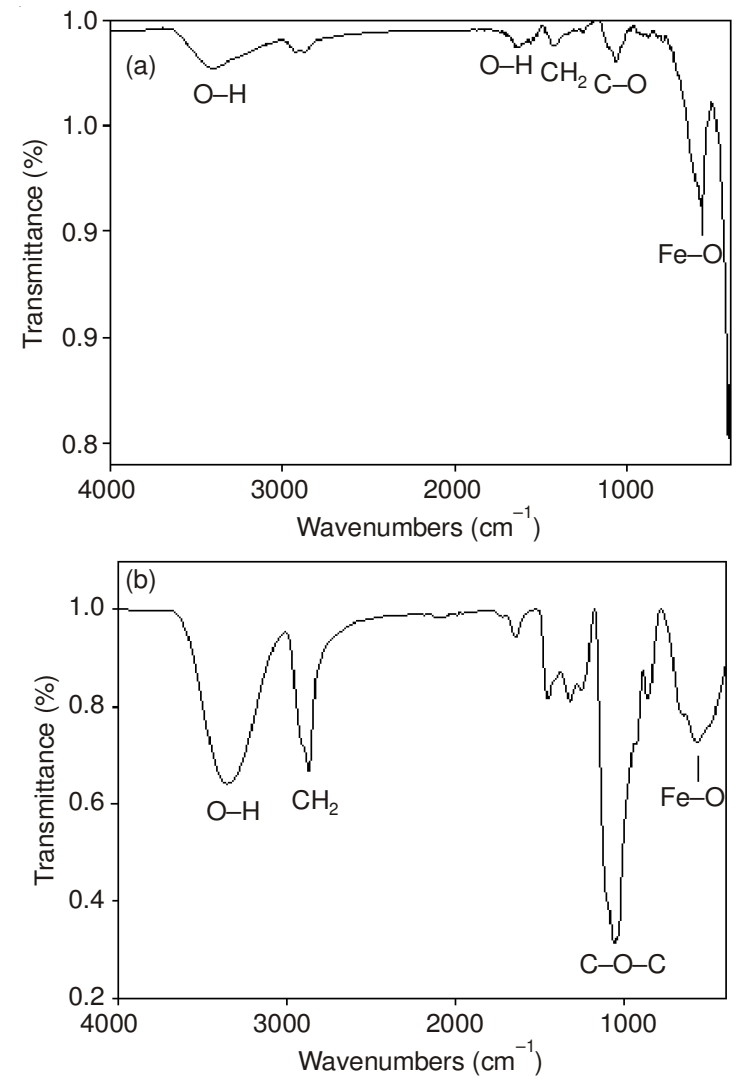

Fig. 4. FT-IR spectra of (a) the as-synthesized MNPs and (b) the HPGMNPs

Fig. 5. (a) Thermogravimetric analysis results and (b) magnetization curves for the as-synthesized MNPs and HPG-MNPs

value) of the as-synthesized MNPs and HPG-MNPs at room temperature were 52.4 and $20.7 \mathrm{emu} \mathrm{g}^{-1}$, respectively. From the TGA results, the normalized saturated magnetization value in the HPG-MNPs $\left(63.6 \mathrm{emu} \mathrm{g}^{-1} \mathrm{Fe}_{3} \mathrm{O}_{4}\right)$ was equivalent to that of the as-synthesized MNPs (63.2 emu g $\left.{ }^{-1} \mathrm{Fe}_{3} \mathrm{O}_{4}\right)$, indicating that the grafting of HPG did not change the magnetic properties of the magnetite core.

The utility of the HPG-MNPs as a draw solution was explored by measuring the osmolality of the HPG-MNPs in water at various concentrations using a Fiske ${ }^{\circledR} 210$ osmometer (Advanced Instruments, Inc.). The osmotic pressures of the draw solutions were 3.9, 10.1 and 15.24 atm for 100, 200 and $300 \mathrm{mg} / \mathrm{mL}$ HPG-MNPs, respectively. These results indicate that the HPG-MNPs are potentially useful as a draw solute in forward osmosis processes. The HPG-MNPs were evaluated as draw solutes in a lab-scale forward osmosis process (Scheme-I). In forward osmosis processes, the water flux may be calculated from the volume change of the feed solution.

$$
\mathrm{Jv}=\frac{\Delta \mathrm{V}}{(\mathrm{A} \Delta \mathrm{t})}
$$

where $\Delta \mathrm{V}(\mathrm{L})$ is the water volume that has permeated the membrane over an interval of time $(\Delta \mathrm{t}, \mathrm{h})$ and $\mathrm{A}$ is the effective membrane surface area $\left(\mathrm{m}^{2}\right)$.

Fig. 6 shows the flux of pure water as a function of draw solute (HPG-MNP) concentration, using water as a feed solution. As the concentration of the draw solution increased, the water flux increased due to an increase in the osmotic pressure from 2.2-4.9 and $6.7 \mathrm{~L} / \mathrm{m}^{2} \mathrm{~h}$ for 100,200 and $300 \mathrm{mg} / \mathrm{mL}$ HPG- 


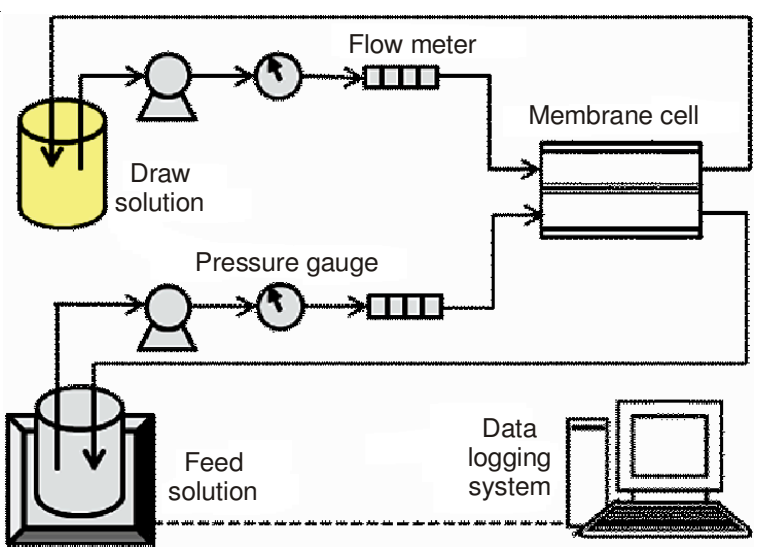

Scheme-I: Diagram illustrating the forward osmosis system

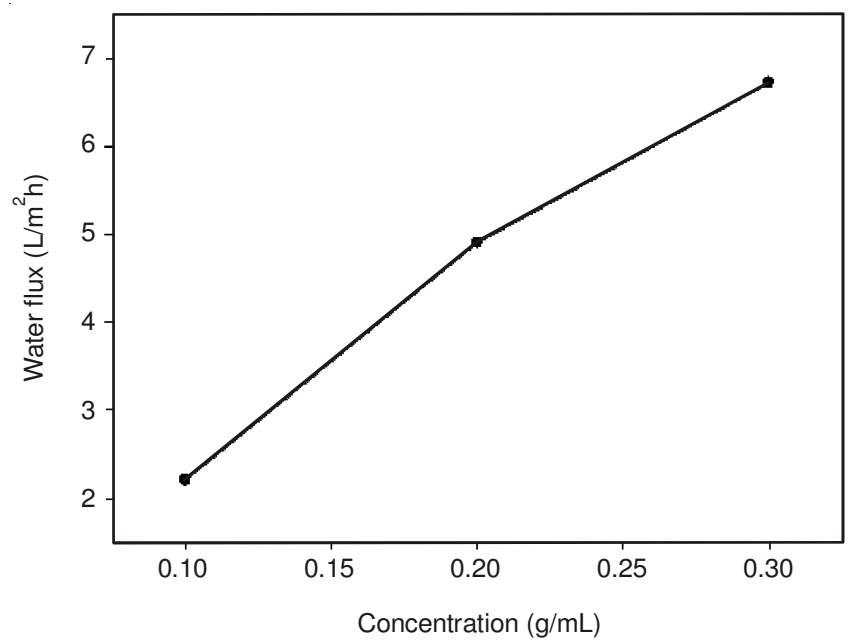

Fig. 6. Pure water flux as a function of the HPG-MNP draw solution concentration

MNPs, respectively. These results demonstrated that the HPGMNPs could draw water across the HTI forward osmosis membrane from the feed solution. Fig. 7 shows HPG-MNPs were present in aqueous phase even after placing the external magnet, indicating that aggregate were not formed due to the high water solubility of HPG on nanoparticle surface. For the reuse of HPG-MNPs as a draw solute, the HPG-MNPs can be easily separated from the water using ultrafiltration techniques without decrease in forward osmosis performance caused by aggregate of MNPs ${ }^{10}$.

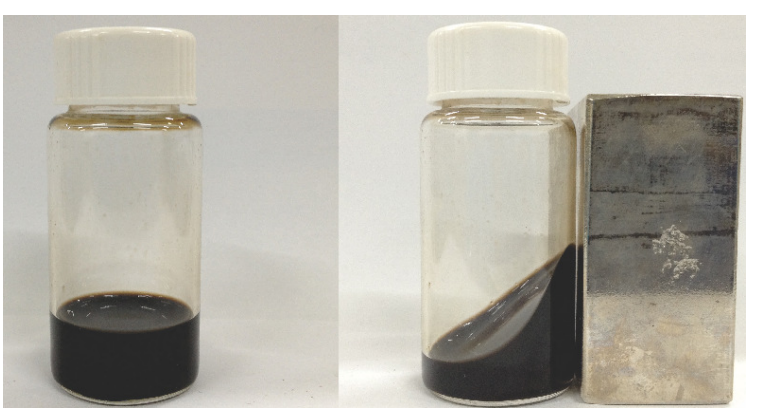

Fig. 7. Photographic image of HPG-MNPs solution with and without a permanent magnet

\section{Conclusion}

In this study, HPG-coated MNPs were successfully synthesized and examined for their utility as a new MNP based draw solute in forward osmosis processes. The HPG-MNPs showed no aggregate of particles in water even after placing external magnet and exhibited a high water flux in forward osmosis process. The HPG-MNPs are, therefore, potentially useful as a draw solute in forward osmosis processes.

\section{ACKNOWLEDGEMENTS}

This work was support by the NRF grant funded by the Korea government (MSIP) (No. 2012M2A8A5025996 and No. NRF-2012M2A8A1030164).

\section{REFERENCES}

1. T.Y. Cath, A.E. Childress and M. Elimelech, J. Membr. Sci., 281, 70 (2006).

2. S. Zhao, L. Zou, C.Y. Tang and D. Mulcahy, J. Membr. Sci., 396, 1 (2012).

3. L. Chekli, S. Phuntsho, H.K. Shon, S. Vigneswaran, J. Kandasamy and A. Chanan, Water Treat., 43, 167 (2012).

4. M.M. Ling, K.Y. Wang and T.-S. Chung, Ind. Eng. Chem. Res., 49, 5869 (2010).

5. Q. Ge, J. Su, T.-S. Chung and G. Amy, Ind. Eng. Chem. Res., 50, 382 (2011).

6. M. Caldero'n, M.A. Quadir, S.K. Sharma and R. Haag, Adv. Mater., 22, 190 (2010).

7. J. Wan, W. Cai, X. Meng and E. Liu, Chem. Commun. (Camb.), 47, 5004 (2007).

8. L. Zhao, T. Takimoto, M. Ito, N. Kitagawa, T. Kimura and N. Komatsu, Angew. Chem. Int. Ed., 50, 1388 (2011).

9. D. Maity and D.C. Agrawal, J. Magn. Magn. Mater., 308, 46 (2007).

10. M.M. Ling and T.-S. Chung, Desalination, 278, 194 (2011). 